\title{
Association of time of day and extubation success in very low birthweight infants: a multicenter cohort study
}

\author{
Brittany Guy ${ }^{1}$, Mary Eva Dye ${ }^{2,3}$, Laura Richards ${ }^{4}$, Scott O. Guthrie iD ${ }^{2,4}$ and L. Dupree Hatch III iD ${ }^{2,5,6}{ }^{凶}$ \\ (C) The Author(s), under exclusive licence to Springer Nature America, Inc. 2021
}

OBJECTIVE: To determine the association of overnight extubation (OE) with extubation success.

STUDY DESIGN: Retrospective cohort study in three NICUs from 2016 to 2020. Infants without congenital anomalies, less than 1500 grams at birth, who were ventilated and received an extubation attempt were included. Primary exposure was OE (7:00 pm-6:59 am) and outcome was extubation success defined as no mechanical ventilation for at least 7 days after extubation. RESULTS: A total of 76/379 (20\%) infants received OE. Infants extubated during the daytime were older and had higher illness severity markers. Extubation success rates did not differ for overnight $(57 / 76,75 \%)$ versus daytime extubations $(231 / 303,76 \%)$ after adjusting for confounders (adjusted relative risk $0.95,95 \% \mathrm{Cl} 0.82-1.11$ ).

CONCLUSION: Though infants in our cohort undergoing daytime and OE were dissimilar, extubation success rates did not differ. Larger multicenter studies are needed to test our findings and identify markers of extubation readiness in preterm infants.

Journal of Perinatology (2021) 41:2532-2536; https://doi.org/10.1038/s41372-021-01168-6

\section{INTRODUCTION}

Mechanical ventilation (MV) is common in preterm infants in the Neonatal Intensive Care Unit (NICU), occurring in up to $85 \%$ of infants less than 29 weeks' gestational age (GA) at birth [1]. Though lifesaving for many infants, the duration of $M V$ is independently associated with increased mortality [2], neurodevelopmental impairment [3-5], and bronchopulmonary dysplasia $[6,7]$. Given these risks, timely extubation from MV is essential for ensuring optimal clinical outcomes for preterm infants receiving $\mathrm{MV}$.

Similar to MV, extubation failure, or reintubation after a failed trial of extubation, is common and associated with adverse outcomes in preterm infants. Extubation failure is reported to occur in up to $40 \%$ of infants born $<28$ weeks' GA [8] and is associated with increased mortality, bronchopulmonary dysplasia, severe intraventricular hemorrhage, and longer duration of MV, length of stay, and supplemental oxygen therapy [8,9]. Given the competing risks of prolonging $\mathrm{MV}$ and extubation failure, neonatal clinicians must strike a balance between minimizing the duration of $M V$ and ensuring the highest chance of successful extubation.

Little is known about the contribution of systems and personnel factors such as staffing and bedside provider experience, which often differ by time of day or shift, on extubation success. Most studies in mechanically ventilated adults have shown that overnight extubations (OEs), those performed from $7 \mathrm{pm}$ to 6:59 am, result in similar rates of extubation success compared to those performed during the day [10-12]. To date, no studies have evaluated the effect of time of day of extubation on success in the NICU. The objective of this study was to determine the association of OE with extubation success in very low birth weight (VLBW) infants.

\section{METHODS \\ Study design/Setting}

We performed a retrospective cohort study in three geographically distinct NICUs within two medical centers. Our cohort included all VLBW infants (birth weight $<1500 \mathrm{~g}$ ) admitted to the NICUs, receiving MV for any period of time, and having at least one planned extubation attempt from May 1 , 2016 to January 31, 2020. We excluded infants with life-limiting congenital anomalies such as congenital diaphragmatic hernia or critical congenital heart disease.

The study was conducted in the Monroe Carell Jr. Children's Hospital at Vanderbilt NICU, the Stahlman NICU at the Vanderbilt University Medical Center (VUMC), and the Jackson-Madison County General Hospital NICU. The Monroe Carell Jr. Children's Hospital at Vanderbilt NICU is a 78-bed, level 4 unit [13] serving inborn infants with complex surgical or cardiac anomalies and outborn infants transported to VUMC from the surrounding catchment area. The Stahlman NICU is a 20-bed unit adjacent to the VUMC labor and delivery unit that primarily houses preterm and ill term inborn infants. The Jackson-Madison County General Hospital NICU is a level 3 [13], 30-bed community NICU located in West Central Tennessee.

\section{Exposure variables}

The primary exposure in our study was $\mathrm{OE}$, defined as a planned extubation attempt occurring during night shift hours (7:00 pm-6:59 am) [14]. For infants with multiple extubation attempts, only the first attempt was evaluated. In all three units, staffing patterns for physicians and advanced practice providers differ between day and night shift though all units have one or more clinicians in-house who are skilled at intubation at

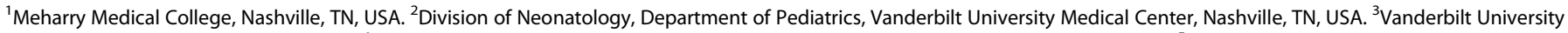

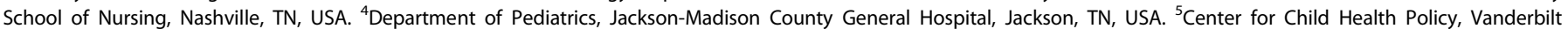
University Medical Center, Nashville, TN, USA. ${ }^{6}$ Critical Illness, Brain Dysfunction, and Survivorship (CIBS) Center, Vanderbilt University Medical Center, Nashville, TN, USA. email: Leon.d.hatch@vumc.org 
all times. Nursing and respiratory therapy staffing does not differ between day and night shift in these units, though nursing and respiratory therapy experience level is generally higher during day shifts. Staffing models for each unit do not differ significantly by day of the week and are provided in Supplementary Methods 1. Extubation timing was determined by the clinical team in each NICU and no formal extubation criteria existed. The usual practice in all three units is to extubate infants less than 32 weeks' postmenstrual age to either non-synchronized nasal intermittent positivepressure ventilation or nasal continuous positive airway pressure after receiving pre-extubation caffeine therapy. Transcutaneous carbon dioxide monitoring is uniformly used in one of the study NICUs and sporadically used in the other two.

\section{Study outcomes}

The primary outcome was successful extubation, defined as being free from mechanical ventilation for 7 or more days after a planned extubation attempt [15]. Unplanned extubations were prospectively monitored in each NICU and these extubations were excluded from analyses. Two infants were electively reintubated within the 7-day period due to planned procedures, one for subgaleal shunt placement and the other for amniotic band release. These infants were excluded from the study cohort. For infants who were reintubated after a planned extubation attempt, we measured the time from extubation to reintubation in minutes as a secondary outcome.

\section{Statistical analysis}

For our pre-specified main analysis, we calculated the relative risk of extubation success after OE compared to those performed during the day in the entire cohort of infants (main cohort) using multivariable modified Poisson regression models [16]. We determined model variables a priori, choosing variables shown to affect extubation success in two previous studies in preterm infants: GA, small for GA status, 5-minute Apgar score, peak supplemental oxygen during the first $24 \mathrm{~h}$, supplemental oxygen at extubation, and postnatal age at extubation $[8,9]$. In prior studies of extubation success, higher blood $\mathrm{pH}$ at the time of extubation has been shown to be a significant predictor of extubation and we planned to include this variable in our models. However, most infants did not have a blood gas within $6 \mathrm{~h}$ of extubation and this variable was not included in the final multivariable model.

For infants who required reintubation in our main cohort, we plotted Kaplan-Meier curves of the time from extubation to reintubation separately by day and night extubations. We also tested the hypothesis that infants extubated at night may fail earlier than those during the day by calculating the Wilcoxon test for equality of the survival curves.

In addition, because of large differences in the populations of patients extubated during the day and overnight, we performed two post-hoc secondary analyses using two different doubly robust causal inference methods. Doubly robust estimators combine outcome regression with sample weighting (e.g., propensity score) such that the estimates of causal effect are robust to potential misspecification of one of the models [17]. First, we calculated propensity scores for all infants in the main cohort and used these scores to calculate inverse probability of treatment weights (IPWs) for the likelihood that an infant would be extubated overnight. Model covariates for IPWs were iteratively chosen to produce an analytic sample with relative covariate balance between day and OEs [18]. We used this cohort (IPW cohort) to calculate the average treatment effect of OE on extubation success by further adjusting for the a priori chosen variables above and those variables with residual imbalance after weighting using the teffects ipwra command in Stata. In our second analysis, we created an analytic sample using entropy balancing (EB). Entropy balancing is a data preprocessing technique used in observational studies with a binary treatment that assigns analytic weights to the control group in order to achieve exact balance of covariate moments with the treatment group [19]. In other words, the control observations were weighted to form an analytic sample that resembles the treatment group. After applying EB to our main cohort, we calculated the relative risk of successful extubation overnight in the EB cohort using multivariable modified Poisson regression. Detailed explanation of our methods including variables used for all models, detailed references, and associated Stata code are found in Supplementary Methods 2. In all cohorts, we calculated descriptive statistics for demographics, clinical characteristics, and outcomes using means and standard deviations for all continuous variables and percentages for nominal variables. We assessed covariate imbalance using standardized differences and variance ratios between overnight and day

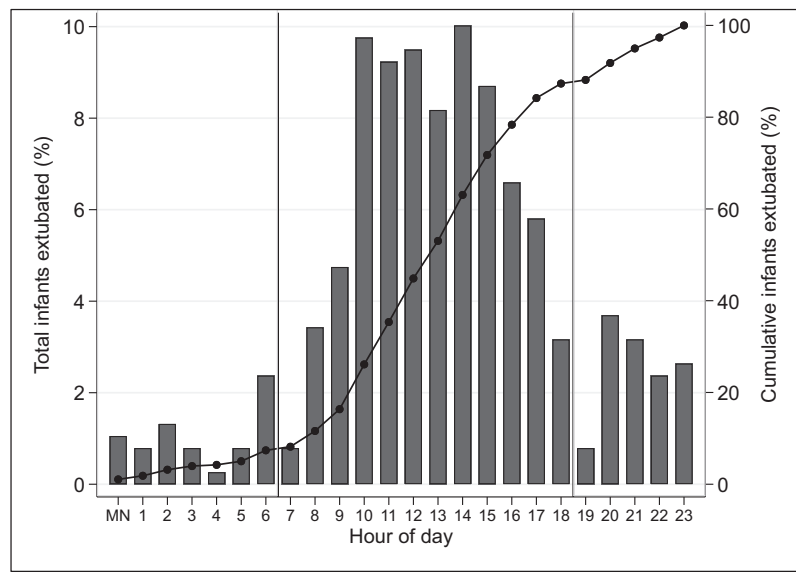

Fig. 1 Timing of extubation attempts by hour of day. Individual bars show the percent of infants extubated within each hour with a low of $0.3 \%$ of extubations from $04: 00$ to $5: 00$ to a high of $10.0 \%$ of extubations from 14:00 to 15:00. Black line shows the cumulative percent of extubation attempts. Extubations during night shift hours accounted for only $20 \%$ (76/379) of all extubation attempts.; MNmidnight.

extubations. Standardized differences compare the difference in means in units of the pooled standard deviation and are not influenced by the sample size. A difference of $\geq 0.1$ in the standardized differences [20] or variance ratios $<0.5$ or $>2.0$ were treated as concerning [21]. We performed one secondary analysis by repeating our main analyses in infants who had their primary extubation attempt within the first 30 days of life.

Study data were housed in the Research Electronic Data Capture program hosted at VUMC. All analyses were completed in Stata v.15.2. All statistical tests were two-tailed and $p$ values $<0.05$ were considered significant. The study received institutional review board approval with a waiver of informed consent at both medical centers.

\section{RESULTS}

\section{Main cohort}

During the study period, 535 VLBW infants received MV in one of the NICUs for 9085 ventilator days. From this population, 379/535 (71\%) infants met study inclusion criteria. The majority of eligible infants $(303 / 379,80 \%)$ received their first extubation attempt during day shift and this was similar across all three study NICUs (77-84\%). The peak timing of extubation attempts in our main cohort was from 10:00 am to 3:00 pm (Fig. 1). Nearly all infants were receiving caffeine at extubation and were extubated to either NIPPV or CPAP. Infants extubated during day shift were significantly different than infants extubated at night. Infants extubated during day shift were more immature at birth (mean GA 26.4 weeks vs. 26.8 weeks), had more severe respiratory disease after birth with higher peak oxygen requirement during the first $24 \mathrm{~h}$ of life (mean $\mathrm{FiO}_{2} 0.48$ vs. 0.41 ) and more use of surfactant, and were older at first extubation attempt (mean postnatal age 20.5 days vs. 14.6 days) (Table 1 ).

In the main cohort, $288 / 379$ (76\%) infants remained free from MV at 7 days following their first extubation attempt. The rate of extubation success did not differ for infants extubated during the day $(231 / 303,76 \%)$ versus overnight $(57 / 76,75 \%)$ with an adjusted relative risk of $0.95(95 \% \mathrm{Cl} 0.82-1.11)$ for OE success. We found no difference in time to reintubation of infants extubated during the day and infants extubated overnight in our main cohort (Fig. 2). The median time from extubation to reintubation in the main cohort was $19.0 \mathrm{~h}(95 \% \mathrm{Cl} 10.1-23.7)$ in day extubations and $25.1 \mathrm{~h}(95 \% \mathrm{Cl} 6.6-31.7)$ in OEs $(p=0.6)$. The majority of infants, 56/91 (62\%) were reintubated during the day. Findings were similar in infants less than 30 days of age at extubation (Table 2). 
Table 1. Clinical characteristics at birth and time of extubation in main cohort.

\begin{tabular}{|c|c|c|c|}
\hline \multirow[b]{2}{*}{ Mean (SD) unless noted } & \multicolumn{3}{|c|}{ Main Cohort $(n=379)$} \\
\hline & Day $(n=303)$ & Overnight $(n=76)$ & Absolute Std. Diff. \\
\hline GA- weeks & $26.4(2.3)$ & $26.8(2.5)$ & 0.15 \\
\hline Small for GA status- $n(\%)$ & $56(18)$ & $19(25)$ & 0.16 \\
\hline Male sex- $n(\%)$ & $159(52)$ & $43(57)$ & 0.08 \\
\hline 5-minute Apgar score ${ }^{a}$ & $5.9(2.4)$ & $5.3(2.3)$ & 0.27 \\
\hline Highest $\mathrm{FiO}_{2}$ in first $24 \mathrm{~h}$ of life $\mathrm{c}^{\mathrm{C}}$ & $0.48(0.23)$ & $0.41(0.24)$ & 0.28 \\
\hline Inborn- $n(\%)$ & $205(68)$ & $55(72)$ & 0.1 \\
\hline Surfactant doses- $n(\%)$ & & & 0.29 \\
\hline 0 & $46(15)$ & $12(16)$ & \\
\hline 1 & $152(50)$ & $51(67)$ & \\
\hline High-frequency ventilation use at extubation- $n$ (\%) & $34(11)$ & $11(14)$ & 0.1 \\
\hline Sedation- $n(\%)$ & $15(5)$ & $4(5)$ & 0.01 \\
\hline Post-extubation respiratory support- $n$ (\%) & & & 0.15 \\
\hline NIPPV & $44(15)$ & $5(7)$ & \\
\hline CPAP & $233(77)$ & $64(84)$ & \\
\hline High-flow cannula & $19(6)$ & $6(7)$ & \\
\hline Low-flow cannula & $3(1)$ & $1(1)$ & \\
\hline Room air & $4(1)$ & $1(1)$ & \\
\hline Postnatal age at extubation- days & $20.5(35.0)$ & $14.6(33.1)$ & 0.18 \\
\hline Postmenstrual age at extubation- weeks & $28.9(4.7)$ & $28.5(4.4)$ & 0.08 \\
\hline
\end{tabular}

CPAP continuous positive airway pressure, $\mathrm{FiO}_{2}$ fraction of inspired oxygen, GA gestational age, $M V$ mechanical ventilation, NIPPV nasal intermittent positivepressure ventilation.

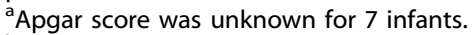

${ }^{b}$ Antenatal steroids was unknown for 15 infants.

${ }^{\mathrm{C}} \mathrm{Highest} \mathrm{FiO}_{2}$ was unknown for 58 infants.

\section{Secondary cohorts}

In the main cohort, $307 / 379$ (81\%) infants had complete covariate data and were considered for the IPW cohort. The majority of excluded infants were outborn infants in whom the peak $\mathrm{FiO}_{2}$ during the first $24 \mathrm{~h}$ of life was unknown $(n=58)$. Propensity scores for OEs in the IPW cohort ranged from 1 to $69 \%$ in infants extubated during the day and $6-74 \%$ for infants extubated overnight. After adjustment, the IPW cohort showed improved covariate balance (Supplementary Table 1); mean imbalance in absolute standardized differences decreased from 0.17 in the main cohort to 0.06 and the number of variables with an absolute standardized difference $\geq 0.1$ decreased from 14 to 3 after IPW weighting. Differences remained in 5-min Apgar scores, days of $\mathrm{MV}$ at extubation, and percent of infants receiving high-frequency ventilation at extubation in the IPW cohort and these variables were included in regression adjustment. A total of 356/379 (94\%) infants in the main cohort were included in the EB cohort. Excluded infants were either missing antenatal steroids history or 5-min Apgar score. In the EB cohort, all covariates showed balance in absolute standardized differences and variance ratios (Supplementary Tables 1 and 2).
In the IPW cohort, 120/160 (75\%) weighted extubations during the day and 107/147 (73\%) of weighted OEs were successful. We calculated an average treatment effect of $0.00(95 \% \mathrm{Cl}-0.12$ to 0.12) for OEs indicating that infants who were extubated overnight had no significant change in chances of remaining extubated at 7 days after weighting and regression adjustment. In the EB cohort, infants extubated overnight had an adjusted relative risk of $0.95(0.78-1.15)$ for extubation success compared to the daytime group.

\section{DISCUSSION}

In this study we report, for the first time, the rates of extubation success in VLBW infants in the NICU based on time of day of extubation. Similar to observational data in mechanically ventilated adults [14], 80\% of extubations in our cohort occurred during the day and this was similar across the study NICUs. We found that rates of extubation success did not differ based on time of day. The rates remained similar even after adjusting for patient characteristics, which differed markedly between the two groups. 
While this is the first study to evaluate the association between time of day and extubation success in the NICU, multiple studies have evaluated this association in critically ill adults and children. In adults, the outcomes of $\mathrm{OE}$ are mixed with the majority of studies showing that $\mathrm{OE}$ is not associated with reintubation or adverse outcomes [10-12, 22]. Several studies in adult patients, primarily those conducted in patients after cardiac surgery, have shown a decreased duration of MV [10] and shorter lengths of stay in patients who underwent $O E$ with no significant increase in mortality [11, 22]. In contrast, one large retrospective cohort study in adult ICU patients found that the rate of mortality was significantly increased in patients undergoing OE [14]. In pediatric patients, no studies have specifically evaluated the effect of $O E$ on outcomes. da Silva et al. performed a single-center, retrospective cohort study in 480 patients in the pediatric ICU that attempted to determine the outcomes of "off-hours" extubations, defined as those that occurred either overnight or on a weekend or holiday. In this study, the rate of reintubation at $24 \mathrm{~h}$ after extubation was similar between the "on-" and "off-hours" groups. These authors found a decrease in the average ICU length of stay in patients in the "off-hours" extubation group with no increase in mortality suggesting a potential benefit to "off-hours" extubation [23].

While we found no association between time of day and extubation success in our cohort, the infants in our study had a relatively high rate of extubation success compared to other reports in preterm infants. In a secondary analysis of 926 infants in the SUPPORT trial, Chawla et al. reported an extubation success rate of $58 \%$ at 5 days post-extubation [8]. Manley et al. reported an

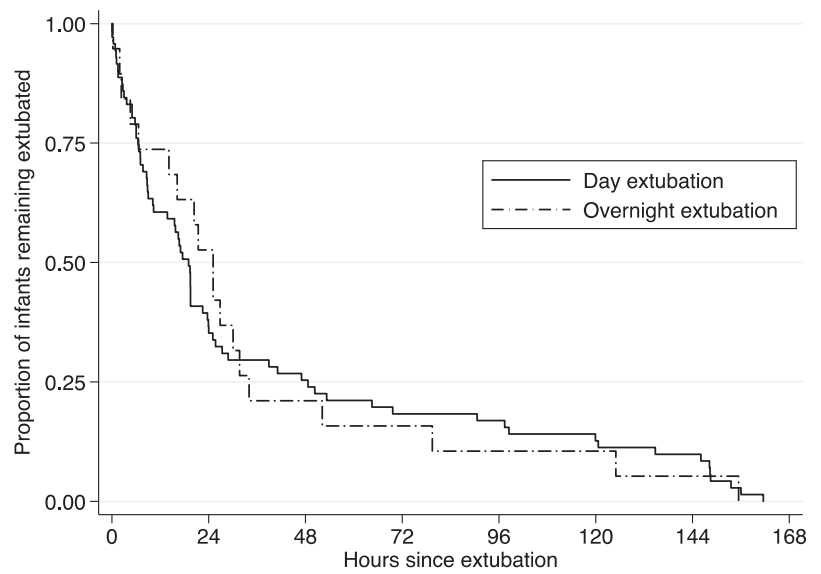

Fig. 2 Kaplan-Meier plot of time to reintubation in infants by timing of extubation attempt. In infants who required reintubation prior to 7 days, there was no significant difference in the time to extubation failure when stratified by time of day with median time to failure of $19.0 \mathrm{~h}(95 \% \mathrm{Cl} 10.1-23.7)$ in day and $25.1 \mathrm{~h}(95 \% \mathrm{Cl}$ $6.6-31.7)$ in overnight extubations $(p=0.6)$. extubation success rate of $68 \%$ at 7 days post-extubation in 174 extremely preterm infants [9] compared to $76 \%$ we observed in our study. In both of these studies, infants were slightly more immature compared to infants in our study. Furthermore, in contrast to the studies by Chawla et al. and Manley et al., the decision to extubate and the decision to reintubate in our study was at the discretion of the clinical team.

One of the main findings in our study was that $80 \%$ of eligible infants had their first extubation attempt during day shift hours, similar to published data in ventilated adults [14]. One potential explanation for this finding may be a bias by clinicians' in the study units for attempting extubation during the day due to a perceived higher chance of success compared to extubation at night. We observed evidence of this in the difference in the clinical characteristics of infants undergoing day and overnight extubations with sicker infants being extubated during the day. Another potential explanation for the large number of day shift extubations is that no formal extubation criteria existed in the three study NICUs. Frontline clinicians may wait until rounds to discuss candidacy for extubation. There is evidence of this in our study given that most extubations follow multidisciplinary rounds ( $10 \mathrm{am}-3 \mathrm{pm})$. In a unit where formal extubation criteria exist and infants are extubated when they meet objective criteria, we would expect infants may be extubated uniformly throughout the day. While the lack of formal extubation criteria is a limitation of our study, it likely reflects prevailing practice in many units, where formal mechanical ventilation guidelines and extubation criteria do not exist [24].

Our study has several additional limitations. First and most importantly, infants undergoing OE were significantly different than those extubated during the day. We attempted to adjust for these differences using multiple statistical methods though none of these techniques are able to account for the possibility that there were unmeasured variables that clinicians used to determine who was extubated overnight. If unmeasured variables existed, our findings may be biased. While a randomized trial would be required to answer the study question without bias, this is likely unethical and only observational studies will be available to answer this important clinical question. Our findings may serve as pilot data for future large multicenter studies that utilize more rigorous causal inference methods such as instrumental variables [25]. Second, though we conducted our study in three NICUs, each with different staffing patterns, our findings may not be generalizable to other NICUs. Caution should especially be taken in generalizing our findings to centers with lower night shift staffing or an inability to safely reintubate infants at night. Third, though we used day and night shift as surrogate measures for staffing, experience, and workload, we did not measure these particular variables. These variables have previously been shown to affect outcomes in the NICU $[26,27]$ and we are not able to say how our results would have differed had these variables been measured and incorporated into analyses. Finally, we did not quantify the specific reasons for the timing of each extubation attempt. It is possible that reasons other than

Table 2. Extubation success by time of day comparing different cohorts.

\begin{tabular}{|c|c|c|c|}
\hline \multirow[b]{2}{*}{ Main cohort } & \multicolumn{3}{|c|}{ Successful extubation $\mathbf{n}(\%)$} \\
\hline & Day & Overnight & Adjusted relative risk $(95 \% \mathrm{Cl})$ \\
\hline All infants $(n=381)$ & $231 / 303(76)$ & $57 / 76(75)$ & $0.95(0.82-1.11)$ \\
\hline Inverse probability of treatment weighted cohort & Day $^{a}$ & Overnight $^{\mathrm{a}}$ & Average treatment effect $(95 \% \mathrm{Cl})$ \\
\hline All infants $\left(n_{\text {weighted }}=307\right)$ & $120(75)$ & $107(73)$ & $0.00(-0.12$ to 0.12$)$ \\
\hline Entropy balancing cohort & Day $^{\mathrm{a}}$ & Overnight $^{a}$ & Adjusted relative risk $(95 \% \mathrm{Cl})^{\mathrm{b}}$ \\
\hline
\end{tabular}

${ }^{a}$ Number and percentage of infants in the following categories are the weighted figures.

${ }^{\mathrm{b}} 95 \%$ confidence intervals $(\mathrm{Cl})$ were estimated through bootstrap replication. 
clinical indications (such as parent availability) were used by clinicians to determine the timing of some extubations.

\section{CONCLUSION}

In summary, most preterm infants in our cohort had their first extubation attempt during the daytime hours with only a minority of infants undergoing OE. Infants extubated during the day were significantly different than infants extubated overnight suggesting that clinicians in the study units are selective about what infants are extubated overnight. Despite this, we found no difference in extubation success rates between day and night shift even after using multiple statistical techniques to account for confounders. While a randomized trial of extubation timing would be unethical, larger, multicenter observational studies using rigorous causal inference methods should be designed to answer this important clinical question and build upon the findings from our study. Given the strong association of MV duration with adverse pulmonary and neurodevelopmental outcomes, evidence-based strategies to identify preterm infants who can safely be extubated, regardless of time of day, are needed.

\section{REFERENCES}

1. Stoll BJ, Hansen NI, Bell EF, Walsh MC, Carlo WA, Shankaran S, et al. Trends in care practices, morbidity, and mortality of extremely preterm neonates, 1993-2012. JAMA. 2015;314:1039-51.

2. Vendettuoli V, Bellu R, Zanini R, Mosca F, Gagliardi L. Italian Neonatal Network. Changes in ventilator strategies and outcomes in preterm infants. Arch Dis Child Fetal Neonatal Ed. 2014;99:F321-4.

3. Laptook AR, O'Shea TM, Shankaran S, Bhaskar B. Neonatal Research Network. Adverse neurodevelopmental outcomes among extremely low birth weight infants with a normal head ultrasound: prevalence and antecedents. Pediatrics. 2005;115:673-80.

4. Guillot M, Guo T, Ufkes S, Schneider J, Synnes A, Chau V, et al. Mechanical ventilation duration, brainstem development, and neurodevelopment in children born preterm: a prospective cohort study. J Pediatr. Published online May 23, 2020. https://doi.org/10.1016/j.jpeds.2020.05.039.

5. Tsai WH, Hwang YS, Hung TY, Weng SF, Lin SJ, Chang WT. Association between mechanical ventilation and neurodevelopmental disorders in a nationwide cohort of extremely low birth weight infants. Res Dev Disabil. 2014;35:1544-50.

6. Jensen EA, DeMauro SB, Kornhauser M, Aghai ZH, Greenspan JS, Dysart KC. Effects of multiple ventilation courses and duration of mechanical ventilation on respiratory outcomes in extremely low-birth-weight infants. JAMA Pediatr. 2015;169:1011-7.

7. Walsh MC, Morris BH, Wrage LA, Vohr BR, Poole WK, Tyson JE, et al. Extremely low birthweight neonates with protracted ventilation: mortality and 18-month neurodevelopmental outcomes. J Pediatr. 2005;146:798-804.

8. Chawla S, Natarajan G, Shankaran S, Carper B, Brion LP, Keszler M, et al. Markers of successful extubation in extremely preterm infants, and morbidity after failed extubation. J Pediatr. 2017;189:113-9.e2.

9. Manley BJ, Doyle LW, Owen LS, Davis PG. Extubating extremely preterm infants: predictors of success and outcomes following failure. J Pediatr. 2016;173:45-9.

10. Everhart KK, Khorsand S, Khandelwal N, Michaelsen KE, Spiekerman CF, Joffe AM. Nighttime extubation does not increase risk of reintubation, length of stay, or mortality: experience of a large, Urban, Teaching Hospital. Anesth Analg. 2019;128:918-23.

11. Tischenkel BR, Gong MN, Shiloh AL, Pittignano VC, Keschner YG, Glueck JA, et al. Daytime versus nighttime extubations: a comparison of reintubation, length of stay, and mortality. J Intensive Care Med. 2016;31:118-26.

12. Krebs ED, Hawkins RB, Mehaffey JH, Fonner CE, Speir AM, Quader MA, et al. Is routine extubation overnight safe in cardiac surgery patients? J Thorac Cardiovasc Surg. 2019;157:1533-42.e2

13. Stark AR. American Academy of Pediatrics Committee on Fetus and Newborn. Levels of neonatal care. Pediatrics. 2004:114:1341-7.

14. Gershengorn HB, Scales DC, Kramer A, Wunsch H. Association between overnight extubations and outcomes in the intensive care unit. JAMA Intern Med. 2016;176:1651-60.
15. Giaccone A, Jensen E, Davis P, Schmidt B. Definitions of extubation success in very premature infants: a systematic review. Arch Dis Child Fetal Neonatal Ed. 2014;99:F124-7.

16. Zou G. A modified poisson regression approach to prospective studies with binary data. Am J Epidemiol. 2004;159:702-6.

17. Funk MJ, Westreich D, Wiesen C, Sturmer T, Brookhart MA, Davidian M. Doubly robust estimation of causal effects. Am J Epidemiol. 2011;173:761-7.

18. Austin PC, Stuart EA. Moving towards best practice when using inverse probability of treatment weighting (IPTW) using the propensity score to estimate causal treatment effects in observational studies. Stat Med. 2015;34:3661-79.

19. Hainmueller J. Entropy balancing for causal effects: a multivariate reweighting method to produce balanced samples in observational studies. Political Anal. 2012;20:25-46.

20. Austin PC. Balance diagnostics for comparing the distribution of baseline covariates between treatment groups in propensity-score matched samples. Stat Med. 2009;28:3083-107.

21. Rubin DB. Using propensity scores to help design observational studies: application to the tobacco litigation. Health Serv Outcomes Res Methodol. 2001;2:169-88.

22. Gershengorn HB, Wunsch $H$, Hua M, Bavaria JE, Gutsche J. Association of overnight extubation with outcomes after cardiac surgery in the intensive care unit. Ann Thorac Surg. 2019;108:432-42.

23. da Silva PS, Reis ME, Fonseca TS, Fonseca MC. Do in-hours or off-hours matter for extubating children in the pediatric intensive care unit? J Crit Care. 2016;36:97-101.

24. Al-Mandari H, Shalish W, Dempsey E, Keszler M, Davis PG, Sant'Anna G. International survey on periextubation practices in extremely preterm infants. Arch Dis Child Fetal Neonatal Ed. 2015;100:F428-31.

25. Cnossen MC, van Essen TA, Ceyisakar IE, Polinder S, Andriessen TM, van der Naalt $\mathrm{J}$, et al. Adjusting for confounding by indication in observational studies: a case study in traumatic brain injury. Clin Epidemiol. 2018;10:841-52.

26. Tubbs-Cooley HL, Mara CA, Carle AC, Mark BA, Pickler RH. Association of nurse workload with missed nursing care in the neonatal intensive care unit. JAMA Pediatr. 2019;173:44-51.

27. Rogowski JA, Staiger D, Patrick T, Horbar J, Kenny M, Lake ET. Nurse staffing and NICU infection rates. JAMA Pediatr. 2013;167:444-50.

\section{AUTHOR CONTRIBUTIONS}

BG and LDH conceptualized and designed the study and wrote the first draft of the manuscript. BG, MED, LR, and SOG performed and oversaw data collection. LDH designed and performed the statistical analyses. BG, MED, LR, SOG, and LDH reviewed and made significant edits to the manuscript and all authors agree with the final version as submitted.

\section{FUNDING}

Use of the Research Electronic Data Capture program (REDCap) was supported by UL1 TR000445 from NCATS/NIH. Mrs. Guy was supported by the Vanderbilt-Meharry James P. Carter Scholars program. Dr. Hatch was supported by the Vanderbilt Department of Pediatrics Turner-Hazinski Research Award and the Gerber Foundation Research Award.

\section{COMPETING INTERESTS}

The authors declare no competing interests.

\section{ADDITIONAL INFORMATION}

Supplementary information The online version contains supplementary material available at https://doi.org/10.1038/s41372-021-01168-6.

Correspondence and requests for materials should be addressed to L.D.H.III.

Reprints and permission information is available at http://www.nature.com/ reprints

Publisher's note Springer Nature remains neutral with regard to jurisdictional claims in published maps and institutional affiliations. 